\title{
Boletín
}

Criminológico

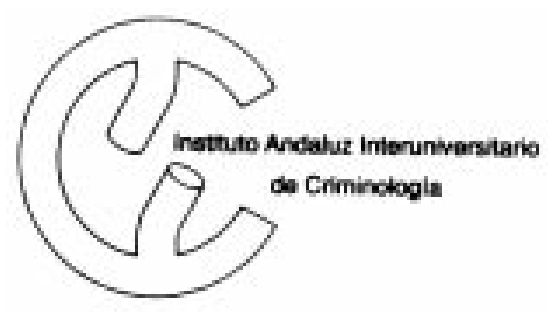

Directora: Elisa García España.

Coordinadora: Juan Carlos Rojo García

Publicado por la Sección de Málaga del IAIC

Edificio Institutos de Investigación, Universidad

de Málaga. Campus de Teatinos, 29071 MALAGA

Tel: (95) 2132325 - Fax: (95) 2132242

Depósito legal: MA 857/1996 ISSN:1137-2427

www . uma . es/estudios/propias/criminologia

Las oficinas de asistencia a víctimas en Andalucía tienen su origen en una propuesta diseñada por el Instituto Andaluz de Criminología, la cual despertó el interés de la Junta de Andalucía y de la Dirección General de Instituciones y Cooperación con la Justicia, cuya Directora, Da Rosa Bendala, instó al IAIC para que concretara la viabilidad de la misma para su puesta en funcionamiento.

Impulsada decisivamente la iniciativa por la Dirección General, el servicio se inicia en 1998 con dos oficinas, una en Málaga y otra en Sevilla, con la intención de ir abriendo paulatinamente otras en las restantes capitales andaluzas y después en todas las grandes ciudades de la Comunidad Andaluza. En estos momentos está ya funcionando la oficina de Granada y, próximamente, se espera el inicio de las de Cádiz y Córdoba.

A partir de un Convenio de Colaboración firmado entre las Universidades andaluzas y la Dirección General, las distintas secciones del IAIC coordinan el servicio en el ámbito de su influencia, financiándose éste gracias a otro Convenio firmado con diversas Cajas de Ahorro.

El propósito final del IAIC y de la Dirección General es que estas oficinas queden integradas como un servicio normalizado dentro del ámbito de actuación de la Administración de Justicia.

\section{PRIMER AÑO DE LA OFICINA DE ASISTENCIA A VÍCTIMAS DE MÁLAGA}

\section{INTRODUCCIÓN .}

El Servicio de Asistencia a Víctimas de Andalucía (SAVA) fue inaugurado en Málaga el 30 de diciembre de 1998. Su primer año de funcionamiento ha sido objeto de una memoria que intenta ser, no una mera divulgación de datos estadísticos, sino un análisis cuantitativo y cualitativo de los mismos,

El objetivo principal del SAVA es ofrecer a las víctimas de un delito o falta la asistencia necesaria para afrontar la situación de desamparo y angustia en la que puedan encontrarse.

\section{ACTUACIONES DEL SAVA}

La ayuda a las víctimas supone una serie de actuaciones de muy diversa índole que va desde el asesoramiento jurídico hasta la atención psicológica y social. Por ello, el equipo que compone el SAVA está integrado por cuatro criminólogos, dos de ellos juristas, uno psicólogo y otro trabajador social, contando además con la colaboración de cinco becarios con iguales titulaciones que los miembros del equipo. La interdisciplinariedad es la fórmula que guía su método de trabajo.

La intervención del SAVA es directa e inmediata, facilitando a la víctima la información y la asistencia precisas para paliar en la medida de lo posible los efectos del trauma sufrido y evitar que sea objeto de posterior victimización (victimización secundaria).

La ubicación del Servicio en el Palacio de Justicia Miramar (junto al Juzgado de Guardia), así como
Autores:

Mf́aría Vicioria Rusas LozanoCarmen García Ferrer María Cheli Shaw Miguel Angel Román Florido

su horario de atención (ininterrumpido desde las 8:30 hasta las 20:00 horas), favorece que la principal fuente de remisión de usuarios sean los Juzgados de Instrucción, especialmente en sus funciones de guardia.

Para prestar una buena asistencia son fundamentales las tareas de gestión, coordinación y derivación realizadas con otros organismos e instituciones, con muchos de los cuales se trabaja en contacto diario, destacando entre ellos los propios Juzgados de Instrucción, la Fiscalía, el Servicio de Atención al Niño, el Instituto Andaluz de la Mujer o los Servicios Sociales comunitarios. Una buena coordinación de todas las instancias implicadas en el tema de la víctima supone una mayor rentabilización de

\section{Boletín Criminológico $N^{o} 46$ Marzo-Abril $2000 \quad$ Página 1}


los recursos y un tratamiento conjunto del problema que, sin duda, repercute en una mejor solución del conflicto y, por tanto, en beneficio de la víctima.

Entre las actividades llevadas a cabo por el SAVA a lo largo de todo el pasado año, no hay que olvidar las de la de difusión del Servicio, a través de numerosas charlas, ponencias, visitas a organismos e instituciones públicas y privadas, material gráfico (carteles y dípticos), artículos publicados, entrevistas en medios de comunicación, etc.

Por último, hay que destacar la reciente puesta en marcha, en febrero de 2000, del programa de guardias de 24 horas para fines de semana y festivos. Cubre un horario que va desde las 20:00 horas del viernes o día anterior al festivo hasta las 9:00 horas del lunes o día posterior al festivo, estando localizable el miembro del equipo que permanece de guardia a través de un teléfono móvil (610 9399 66) y acudiendo en caso de necesidad al lugar donde se demande la asistencia.

\section{LOS USUARIOS DEL SERVICIO}

El número de atenciones realizadas durante el año 1999 ascendió a 1494, de las que casi las dos terceras partes corresponden a asistencias realizadas por vez primera y el resto a seguimiento de casos. Se abrieron 358 expedientes de víctimas y se atendieron a un total de 392 personas que, aun no siendo víctimas de ningún delito o falta, demandaron información y ayuda en otras materias distintas de la penal.

El $78 \%$ de las víctimas que acudieron al SAVA fueron mujeres, porque ellas disponen de más flexibilidad de horario (la mayoría amas de casa y/o paradas) y porque la mujer/madre/ esposa sigue asumiendo el rol de las cuestiones domésticas, siendo ella la que acude a buscar ayuda aunque a veces los afectados sean otros miembros de la familia.

En cuanto a la edad, el intervalo más destacado es entre 31 y 45 años, que es precisamente la etapa más activa en todos los ámbitos de la vida familiar, social y laboral $\mathrm{y}$, por tanto, es cuando la persona es más susceptible de ser objeto de conflictos. (Véase el gráfico 1)

Gráfico 1: EDAD DE LAS VÍCTIMAS

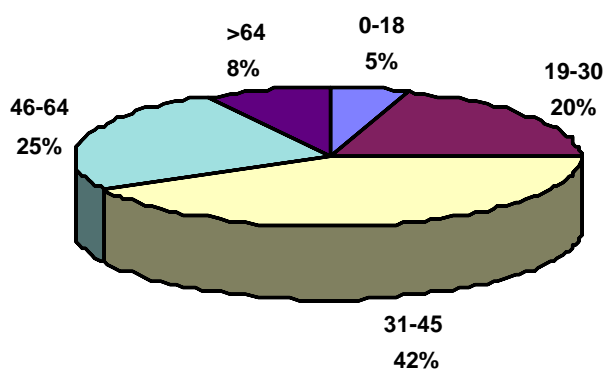

Siguiendo con el perfil de las víctimas, el $47^{\prime} 5 \%$ de las mismas convivían con una pareja (el $43 \%$ estaban casadas y el resto eran parejas de hecho), el $60 \%$ tenía estudios primarios y entre parados y amas de casa sumaban un $36 \%$ (frente al 34\% de personas en activo). Es fácil pensar que personas con alto nivel adquisitivo y/o cultural no suelan acudir a servicios públicos porque pueden acceder a otros privados (Véase el Boletín Criminológico $\mathrm{n}^{\mathrm{o}} 44$ sobre la personación de víctimas en el proceso penal). En este punto, la existencia del SAVA como servicio público y gratuito al alcance de todos adquiere su máxima justificación.

Por lo que se refiere al domicilio de la víctima, hay que destacar el Distrito de Carretera de Cádiz (22\% de los casos), seguido del Distrito Centro (13\%) y de Cruz de Humilladero (11\%). Estos Distritos coinciden con los más poblados de la capital malagueña, con lo que entendemos que la población victimaria es proporcional a la que habita en ellos. (Véase el gráfico 2)

El lugar de comisión de los hechos vino a coincidir en porcentajes y distritos con los del domicilio de la víctima.

En cuanto a datos de victimización, el 83'5\% de las víctimas denuncia los hechos, normalmente después de pasar por el SAVA y ser debidamente asesorado acerca de los derechos que le asisten, de las consecuencias jurídicas de la denuncia, de la posibilidad de solicitar el beneficio de justicia gratuita en muchos casos, del procedimiento, etc. Influyó además el ofrecimiento de acompañamiento por alguien del Servicio a cualquier diligencia que pudiera practicarse, incluido el juicio, siempre que la víctima lo requiriera. Toda esta información y apoyo hace que el denunciante se sienta conocedor de la situación y partícipe en su proceso de normalización. 
La mayoría de los perjuicios sufridos por la víctimas han sido psíquicos (en un $42 \%$ de los casos), si bien lo normal es que confluyan en la misma persona distintos tipos de perjuicios (psíquicos, físicos, económicos o morales).

El 39\% de las víctimas era pareja o ex pareja del autor del delito/falta y en un $20 \%$ de los casos tenía otra relación familiar (en casi todos los de abusos sexuales a menores se daba esta relación); asímismo la frecuencia de victimización resultó ser continuada en el $65 \%$ de los supuestos.

Finalmente, en cuanto a la tipología delictiva, se presentó muy variada. Dentro de las faltas (344 casos) destacaron las cometidas contra las personas (93\%). Con relación a los delitos, de las 155 víctimas habidas, 28 lo fueron de delitos contra la libertad sexual (menores en muchos casos), 24 de delitos contra el patrimonio, 19 de impago de pensión alimentaria, 19 de violencia doméstica y las demás de otros muy variados.(Véase el gráfico 3)
De todos los datos analizados, sería conveniente destacar la relación que se puede apreciar entre distintas variables estudiadas, como puedan ser el sexo, la relación víctima-autor, el domicilio de la víctima, el lugar de la comisión, la frecuencia de victimización, los perjuicios causados y la tipología delictiva.

El que los mismos aparezcan correlacionados responde al hecho de que la mayoría de las víctimas que han pasado por el SAVA han sido por delitos o faltas cometidos normalmente en el seno familiar o vecinal: son víctimas que arrastran unas situaciones muy deterioradas y que acaban acudiendo al Juzgado de Guardia, pensando que el Juez es el único capaz de solucionar su conflicto. Por ello, la mayoría de estos usuarios prefiere acudir directamente al Juzgado de Guardia en lugar de a la Comisaría, siendo finalmente atendidos en el Servicio. Se aprecia también aquí la relación que existe entre aquellas variables y la de la fuente de remisión, dado que, como ya se dijo, la principal fuente era la judicial. Esto constata que el SAVA tiene la asignatura pendiente de conseguir que las Comisarías deriven a las víctimas que acuden allí a denunciar y que, según las estadísticas policiales, en más del $80 \%$ se trata de delitos contra la propiedad.

Los casos de violencia de género que se vieron en el SAVA fueron a menudo derivados por el Instituto Andaluz de la Mujer, organismo éste con el que se trabaja en perfecta coordinación, con remisión bilateral de muchas usuarias al objeto de aprovechar los recursos que cada uno puede ofrecer.

Con relación a las demandas de personas que, aun no siendo víctimas de delito/falta, pasaron por el Servicio, casi el $50 \%$ fueron civiles (especialmente cuestiones de Derecho de Familia). Se han de destacar también los casos derivados de problemas de salud mental y toxicomanías, en los que la respuesta legal no es suficiente, denotándose una carencia de recursos sociales en esta materia.

Gráfico 2: DOMICILIO DE LA VÍCTIMA

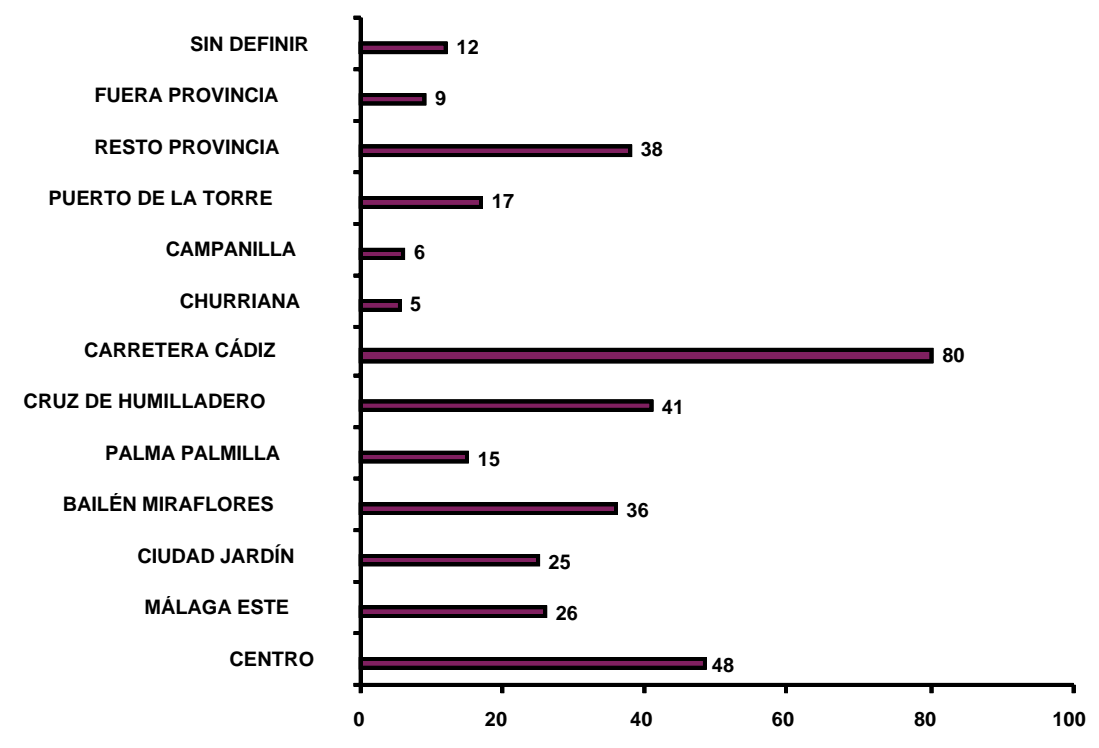

Boletín Criminológico $N^{o} 46$ Marzo-Abril $2000 \quad$ Página 3 


\section{PROYECTOS DEL SAVA}

En cuanto a los proyectos, el SAVA de Málaga tiene en curso uno de prevención de victimización y otro de mediación penal.

Con relación al primero, se pretende llevarlo a cabo a través de asociaciones de vecinos, Institutos, Colegios, Centros Asistenciales de la mujer, Centros de tercera edad, etc., al objeto de difundir las funciones del Servicio y las medidas de prevención contra determinados delitos y/o faltas que afecten a grupos de población más concretos. De hecho, se dieron charlas en colaboración con los Servicios Sociales en todos los Distritos de Málaga.

Por lo que se refiere a la mediación penal entre víctima-autor, hay que señalar cómo la idea de la reparación y de conciliación víctima-delincuente se va extendiendo internacionalmente cada vez más, especialmente con los delincuentes jóvenes y los primarios, como solución que satisface tanto a la víctima, por la pronta reparación del daño que se le ha causado, como al delincuente, que puede verse favorecido bien por el sobreseimiento del proceso cuando sea posible, bien ,cuando no lo sea, por la aplicación de la atenuante de reparación del daño del art. $21.5^{\circ} \mathrm{CP}$,o por la suspensión o la sustitución de la ejecución de la pena privativa de libertad, según el caso, instituciones estas últimas en las que la audiencia de la víctima tiene un papel destacado (arts 80,86,87 y 88 ).

En definitiva, la intervención del SAVA se dirige a propiciar las actitudes de cuantas personas intervienen en la causa y a intentar aunar voluntades en interés de todos: de la víctima, que se va a sentir reparada y considerada; del delincuente, que será favorecido en la medida de lo posible; y de la Administración de Justicia, que puede ver solucionados muchos conflictos con rapidez y eficacia.
Gráfico 3: TIPOLOGÍA DELICTIVA

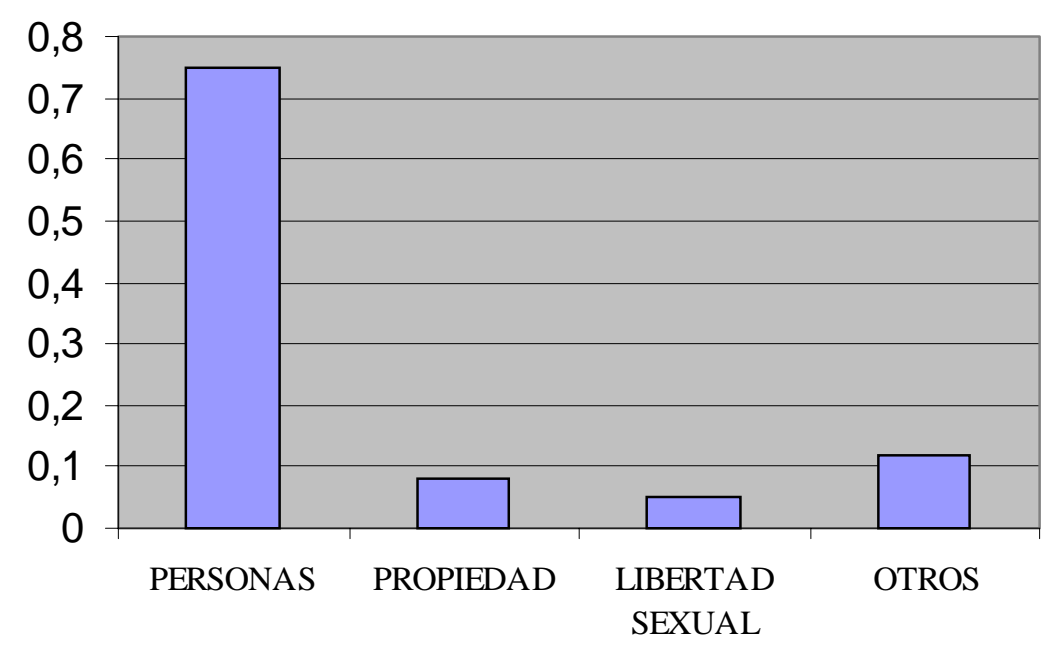

Los principios básicos aplicables al proceso mediador serían los siguientes:

- estando su origen dentro del movimiento de atención y compensación a la víctima, se pretende mediar en principio en aquellos casos en los que exista una víctima individualizada. Sin embargo, en ocasiones, aun cuando la víctima es desconocida cabe la mediación vía reparación a la comunidad (trabajo en beneficio de la comunidad, sometimiento a tratamiento de desintoxicación,etc.);

- no se pretende una finalidad reeducativa del delincuente aunque sí fomentar el sentido de la responsabilidad por lo que ha hecho.

- es imprescindible contar con la libre voluntad de las partes en conflicto

- se busca conseguir una mejora del clima social; se trata de encontrar una solución rápida y eficaz al conflicto, que satisfaga a la víctima y permita al delincuente obtener algún tipo de beneficio por el esfuerzo que le supone reparar. La lentitud de los procedimientos es uno de los factores que más ha ahondado en el desaliento y la desconfianza de los ciudadanos en la Administración de Justicia, y no son pocas las ocasiones en las que dicho procedimiento acaba sin que la víctima haya sido siquiera oída en el juicio ni por supuesto indemnizada;

- aunque la mediación supone una solución extrajudicial al conflicto, debe ubicarse siempre dentro del marco del Derecho Penal, sometida a sus principios generales. Normalmente, el contenido del acuerdo entre las partes conformaría el objeto del juicio, con el visto bueno del Juez sobre la legalidad de los hechos;

- la mediación pasa por que el delincuente se reconozca culpable antes de intervenir;

- la mediación no tiene que implicar necesariamente el enfrentamiento cara a cara de la víctima con el delincuente. Sólo se debe producir en los casos en que se ve conveniente y las partes estén dispuestas a ello. En otro caso, cabe llevarla a efecto a través de sus respectivas defensas. 